\title{
Identifying prognosis and metastasis-associated genes associated with Ewing sarcoma by weighted gene co-expression network analysis
}

\author{
BEN WANG ${ }^{1 *}, \mathrm{JIE} \mathrm{LI}^{2 *}, \mathrm{XIN} \mathrm{LI}^{3 *}$ and YUNSHENG OU ${ }^{1}$ \\ Departments of ${ }^{1}$ Orthopedics, ${ }^{2}$ Oncology and ${ }^{3}$ Respiratory and Critical Care Medicine, \\ The First Affiliated Hospital of Chongqing Medical University, Yuzhong, Chongqing 400016, P.R. China
}

Received February 20, 2019; Accepted June 25, 2019

DOI: $10.3892 / \mathrm{ol} .2019 .10681$

\begin{abstract}
Ewing sarcoma (ES) is a highly malignant pediatric tumor with a low survival rate and a high rate of metastasis. However, there have been limited reports on the exploration of new biomarkers of ES. Therefore, the aim of the present study was to identify the potential hub genes associated with overall vital survival (OVS) and metastasis in ES. Traditional methods for identifying differentially expressed genes lack the in-depth information of mechanistic studies. In this study, a weighted co-expression network for ES was constructed through weighted gene co-expression network analysis to identify co-expression modules associated with clinical phenotypes. The hub genes in the metastasis- and OVS-related co-expression modules were extracted, and the association between the hub genes and patient OVS was verified in another independent Gene Expression Omnibus dataset. Functional annotations and protein-protein interaction analysis of co-expression modules were also used to understand the potential regulatory mechanisms. The results of the functional enrichment analysis revealed that the OVS-associated module was mainly enriched in the cell cycle and immune response, and the metastasis-associated module was enriched in metabolism. A total of four genes (proteasome subunit $\alpha 4, \mathrm{~L} 1$ cell adhesion molecule, serine/threonine kinase receptor-associated protein and cytotoxic T-lymphocyte-associated protein 4) in the OVS-related module and two genes (calcium voltage-gated channel auxiliary subunit $\gamma 2$ and $\gamma$-aminobutyric acid type B receptor subunit 2) in the metastasis-related module were selected as hub genes. Further research on the hub genes
\end{abstract}

Correspondence to: Dr Yunsheng Ou, Department of Orthopedics, The First Affiliated Hospital of Chongqing Medical University, 1 Yixueyuan Road, Yuzhong, Chongqing 400016, P.R. China

E-mail: ouyunsheng2001@163.com

*Contributed equally

Key words: prognosis, Ewing sarcoma, weighted gene co-expression network analysis, metastasis, protein-protein interaction identified in the present study may contribute to the understanding of the mechanism of ES metastasis and progression.

\section{Introduction}

Ewing sarcoma (ES) is a highly malignant tumor with a low survival rate and high rate of metastasis. In a British cohort of patients with ES, ES accounts for $14 \%$ of pediatric bone tumors (1). Comprehensive strategies, including localized surgery, radiotherapy and chemotherapy, have been developed for the treatment of patients with ES (2). However, 30-40\% of patients develop recurrence or metastasis after comprehensive therapy (3). The 5-year survival rate of patients with ES was only $55 \%$ in a British cohort of patients (1). Therefore, further research on the pathogenesis of ES is required to improve the prognosis of affected patients.

According to a previous study, the Ewing sarcoma breakpoint region 1 (EWS)-E26 transformation-specific (ETS) fusion gene is a major factor in ES (4). EWS-ETS fusion genes may confer upon tumors the capacity for metastasis and invasion by altering RNA transcriptional regulation and epigenetic modification $(5,6)$. Previous studies on the EWS-ETS fusion gene have revealed the unique role of this fusion gene in the development of ES; however, treatment targeting the EWS-ETS fusion gene has been difficult to achieve, and there is increasing evidence demonstrating that EWS-ETS may not be the sole driver in metastatic ES (7-9). In addition, the prognostic value of the EWS-ETS fusion gene in patients with ES remains unclear. A retrospective study identified a significant association between EWS-friend leukemia virus integration 1 (FLI1) transcript subtypes and patient outcomes (10). However, two prospective studies were unable to validate this observation $(11,12)$. Consequently, recognition of new therapeutic or predictive biomarkers to contribute to the treatment of ES is urgently needed to improve the prognosis of these patients.

Weighted gene co-expression network analysis (WGCNA) is an advanced approach to studying the associations between genes and clinical traits. Compared with traditional microarray analysis methods, WGCNA analysis uses a soft threshold instead of the hard threshold of traditional differential gene screening, which facilitates the screening of valuable genes 
with small fold changes that may be important in the gene regulation cascade (13).

In addition, WGCNA is an efficient method of studying the associations between genes and clinical phenotypes (14). In the present study, WGCNA was used to classify genes with similar expression patterns into different modules. Associations between the co-expression modules and clinical features of patients were analyzed. The most relevant modules to overall vital survival (OVS) and metastasis status of patients with ES were selected for further analysis.

\section{Materials and methods}

Data collection and preprocessing. ES data were downloaded from the Gene Expression Omnibus (GEO) database (https://www.ncbi.nlm.nih.gov/geo/) as dataset GSE17679 (15). Probes were mapped to gene symbols using the hgu133plus2. $\mathrm{db}$ (version 3.2.3) package (http://bioconductor.org/packages/hgu133plus2.db/) in R. If multiple probes were mapped to gene symbols, the mean value was regarded as the expression value of the gene and the repeated probes were dismissed.

Construction of a weighted co-expression network. The WGCNA package in $\mathrm{R}$ is a widely used method of identifying co-expression networks (16). Pearson's correlation coefficient was calculated to construct the correlation matrix. A soft-thresholding function was used to transform the correlation matrix into a weighted adjacency matrix. To acquire a co-expression network with a balance between scale-independence and mean connectivity, the scale-independence and mean connectivity were calculated in different powers by the soft-connectivity algorithm. The adjacency matrix was transformed into a topological overlap matrix (TOM). 1-TOM was used as the distance measurement to cluster genes into co-expression modules with a deep split value of 2 , minimum size cutoff of 20 and maximum module size of 5,000. A merge height of 0.3 was set as a criterion to cluster similar modules. To identify the association between a co-expression module and a clinical feature, P-values and correlation coefficients were calculated to produce trait-module heatmaps.

PPInetwork construction and functional annotation. The Search Tool for the Retrieval of Interacting Genes/Proteins (STRING) database (date of access, August 2018; https://string-db.org) was used to construct the protein-protein interaction (PPI) network, which contained experimental and in silico predicted PPI information. The data source was set to only the experiment source. Low-connection proteins were hidden. Hub genes are genes that serve a key role in the regulation of the network. In the present study, the betweenness centrality (BC) was used to measure the importance of a node in the PPI network. Cytohubb in Cytoscape software (version 3.7.1, http://www.cytoscape.org) was used to screen for the hub genes, and the top 50 genes with high $\mathrm{BC}$ values were selected for subsequent analysis. Gene Ontology (GO) and Kyoto Encyclopedia of Genes and Genomes (KEGG) functional enrichment analysis was performed using the Metascape website (date of access, August 2018; http://metascape.org).

Survival analysis. Since ES is a rare cancer, after carefully searching the GEO database, ArrayExpress (https://www.ebi. ac.uk/arrayexpress/) and TCGA database (https://cancergenome.nih.gov/) with loose screening criteria (including clinical information), only one GEO dataset, GSE63157 (17), met the requirements (detailed clinical information) of the present study. Following annotation, the expression profiles of 85 samples and 17,186 probes were obtained. The Survival and Survminer packages in $\mathrm{R}$ were used to analyze and visualize the survival data and $\mathrm{P}<0.05$ was considered to indicate a statistically significant difference. All the R packages used in the present study can be found at http://www.bioconductor.org or https://cran.r-project.org/web/packages.

\section{Results}

Construction of weighted gene co-expression network. The expression matrix and clinical data of GSE17679 (15) were downloaded from the GEO database. The scale-independence and mean connectivity were calculated at different thresholds (Fig. 1). Appropriate soft-thresholding has a relatively good balance between scale-independence and mean connectivity of the weighted co-expression network; scale-independence $>0.85$ and average connectivity $<100$ were used as the criteria for a suitable soft threshold. As presented in Fig. 1, a soft threshold power of 5 was chosen for the identification of co-expression modules. A cluster dendrogram based on the dissimilarity of the topological overlap matrix was generated (Fig. 2). Acceptable discriminability was present between each module in the similarity heatmap plot (Fig. 3).

Identification of metastasis-related co-expression modules and OVS-related co-expression modules. The first principal component of each module was defined as the module eigengene (ME), which represented the expression levels of all genes in the module. Gene significance (GS) was used to evaluate the correlation between genes and clinical features by linear regression; the module significance (MS) was calculated by averaging the absolute GS values of all genes in the co-expression module. The most statistically significant module $(\mathrm{P}<0.0001)$ in each clinical feature (OVS and metastasis) were further analyzed.

As presented in Fig. 4, OVS was significantly correlated with the green module $\left(\mathrm{r}^{2}=0.56 ; \mathrm{P}=4 \times 10^{-4}\right)$. The royal blue module was correlated with event-free survival (EFS) $\left(r^{2}=0.5\right.$; $\mathrm{P}=0.002)$, whereas the green-yellow module was most significantly correlated with metastatic state $\left(\mathrm{r}^{2}=0.67, \mathrm{P}=9 \times 10^{-6}\right)$. Subsequent analyses focused on the metastasis-associated co-expression module (yellow-green) and the OVS-associated co-expression module (green).

PPI network in co-expression modules. Following analysis of the STRING database, two PPI networks of the top $50 \mathrm{BC}$ genes in the green and green-yellow modules were constructed. The PPI network of the green module is presented in Fig. 5; the top 10 genes of the green module by $\mathrm{BC}$ value were proteasome subunit $\alpha 4$ (PSMA4; BC=12,145), G-protein subunit $\gamma 5$ ( $\mathrm{BC}=11,152)$, clathrin light chain $\mathrm{A}(\mathrm{BC}=8,382)$, ring-box 1 ( $\mathrm{BC}=7,332)$, ribophorin $\mathrm{II}(\mathrm{BC}=7,288)$, chaperonin-containing TCP1 subunit $6 \mathrm{~B}(\mathrm{BC}=6,931)$, serine/threonine kinase receptor-associated protein (STRAP; $\mathrm{BC}=6,736)$, 3 -phophoinositide-dependent protein kinase $1(\mathrm{BC}=6,652), \mathrm{L} 1$ 

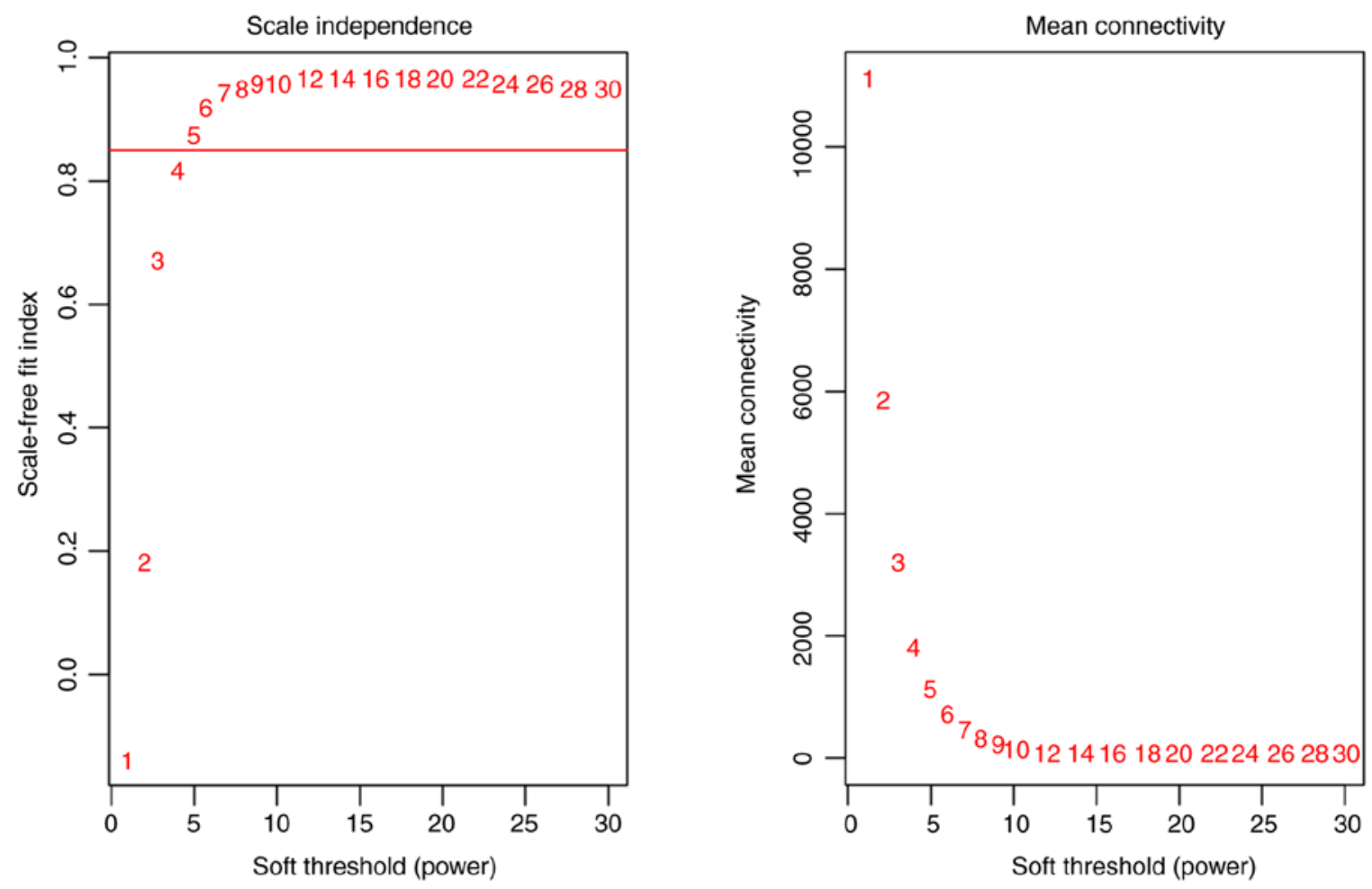

Figure 1. Scale-independence and mean connectivity of the network in different soft-threshold powers. The left panel displays the correlation of soft threshold with scale-free fit index. The right panel displays the influence of soft-threshold power on mean connectivity.

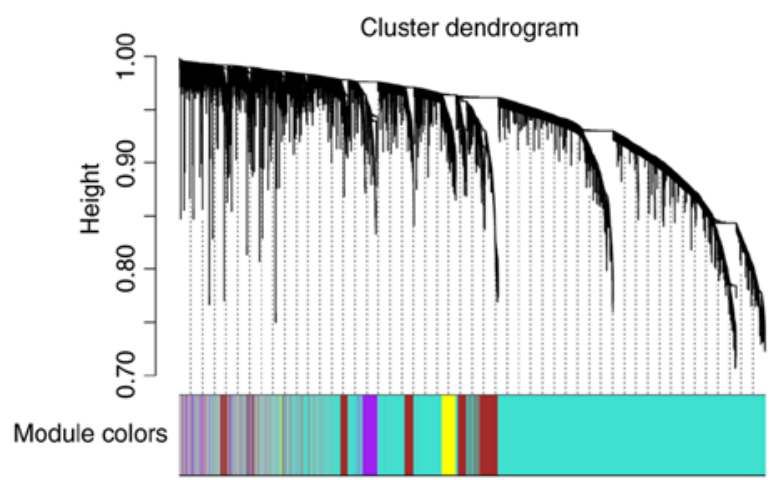

Figure 2. Cluster dendrogram based on the dissimilarity of the topological overlap matrix. The different colors correspond to the co-expression modules in Ewing sarcoma.

cell adhesion molecule (L1CAM; BC=6,468) and Janus kinase $1(\mathrm{BC}=6,346)$. The PPI network of the green-yellow module is presented in Fig. 6. The top 10 genes in green-yellow module by $\mathrm{BC}$ value were vascular endothelial growth factor $\mathrm{D}$ $(\mathrm{BC}=300)$, serpin family A member $1(\mathrm{BC}=252), \mathrm{G}$-protein subunit $\alpha$ transducing $3(\mathrm{BC}=229)$, glutamate ionotropic receptor AMPA subunit $1(\mathrm{BC}=200)$, Met proto-oncogene ( $\mathrm{BC}=198)$, leucine-rich repeat kinase $2(\mathrm{BC}=160)$, fibroblast growth factor receptor $2(\mathrm{BC}=154)$, calcium voltage-gated channel auxiliary subunit $\gamma 2(\mathrm{CACNG} 2 ; \mathrm{BC}=136)$, phospholipase $C \beta 1(B C=112)$ and $\gamma$-aminobutyric acid type $B$ receptor subunit $2(\mathrm{GABBR} 2 ; \mathrm{BC}=94)$.

Functional enrichment of metastasis-and OVS-associated modules. Functional enrichment analysis was used to determine the biological significance of the top 50 high-BC genes in the OVS- and metastasis-associated modules (Table I; Fig. 7). The results demonstrated that the OVS-associated module was mainly enriched in the cell cycle and immune response, including 'adaptive immune system' [ $\log 10(\mathrm{P})=-10.45]$, 'pathways in cancer' $[\log 10(\mathrm{P})=-7.99]$, 'cell cycle, mitotic' [ $\log 10(\mathrm{P})=-7.81]$, 'PID TGFBR PATHWAY' [ $\log 10(\mathrm{P})=-6.92]$, 'L1CAM interactions' $[\log 10(P)=-6.67]$ and 'cell cycle' $[\log 10(\mathrm{P})=-6.57]$.

The enrichment analysis of the top 50 high-BC genes of the metastasis-associated module was performed using Metascape (Table II; Fig. 8); the genes in this module were mainly associated with cellular metabolism and internal signal transduction, including 'defective CSF2RB causes pulmonary surfactant metabolism dysfunction 5 (SMDP5)' [ $\log 10(\mathrm{P})=-14.69]$, 'PI3K-Akt signaling pathway' $[\log 10(\mathrm{P})=-8.59]$, 'metabolism of xenobiotics by cytochrome P450' $[\log 10(\mathrm{P})=-7.91]$, 'Ras signaling pathway' $[\log 10(\mathrm{P})=-6.26]$, 'retinol metabolism' $[\log 10(\mathrm{P})=-4.93]$ and 'neurotransmitter receptors and postsynaptic signal transmission' $[\log 10(\mathrm{P})=-4.68]$.

Prognostic significance of the identified genes. To determine the association between the identified hub genes and OVS of patients, the GEO GSE63157 dataset was used. All the genes in each module were sorted according to $\mathrm{BC}$ value, and $\mathrm{R}$ was used to calculate the association between the total survival time of patients with ES, and the top 10 hub genes ranked by $\mathrm{BC}$ were assessed. The top hub genes ranked by $\mathrm{P}$-value of survival analysis are shown in Fig. 9. Significant associations were observed between the OVS of patients with ES and the four genes (PSMA4, L1CAM, STRAP and CTLA) (all $\mathrm{P}<0.05$; Fig. 9). The association between the top 10 hub genes 
A Eigengene adjacency heatmap

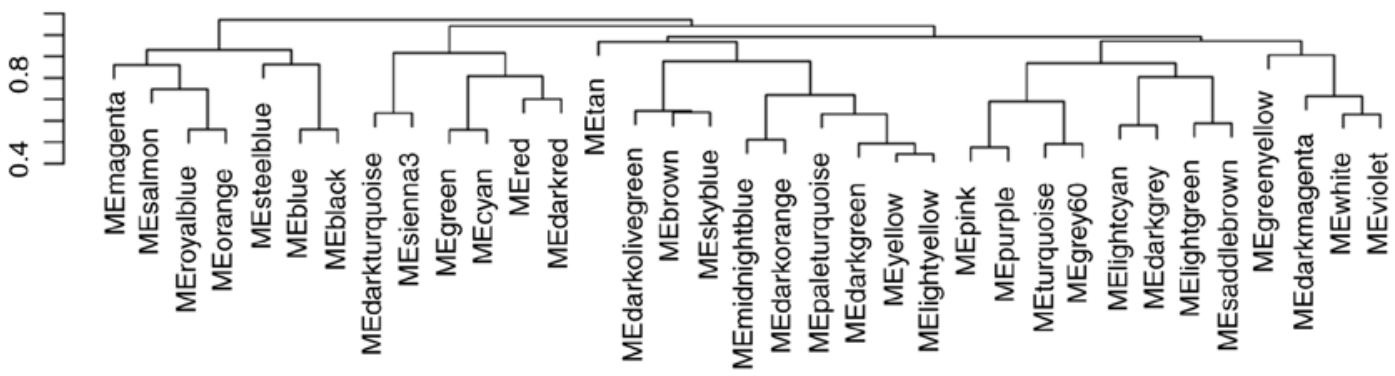

B

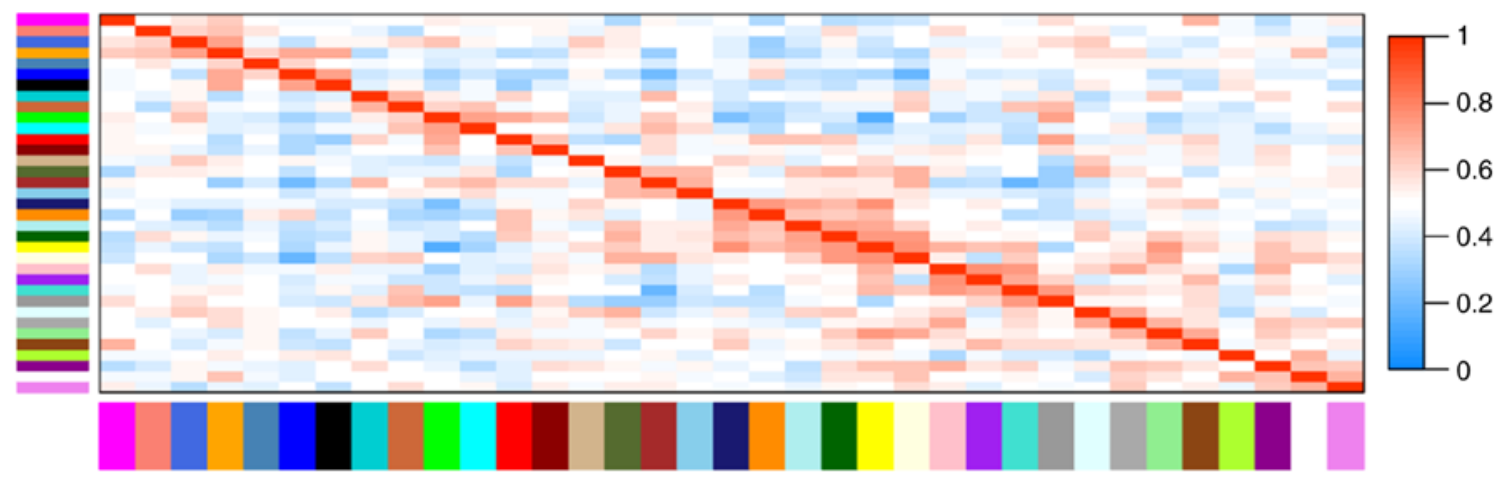

Figure 3. Associations among modules. (A) Hierarchical clustering of module eigengenes that summarize the modules identified in the weighted gene co-expression network analysis. The branches of the dendrogram grouped together indicate a positive correlation. (B) The eigengene adjacency heatmap. Each row and column in the heatmap corresponds to a module (labeled with colors). Red, high adjacency; blue, low adjacency. Red squares along the diagonal axis represent meta-modules.

ranked by $\mathrm{BC}$ ( 2 genes were excluded as the probeID in the GeneChip ${ }^{\circledR}$ platform was unavailable) of the metastasis-related module and the OVS of the patients were also examined. It was determined that two genes were statistically significant prognostic factors for ES. High expression of CACNG2 and low expression of GABBR2 were favorable prognostic factors for patients with ES (Fig. 10).

\section{Discussion}

ES is the second most common pediatric bone tumor (18). Although a comprehensive treatment plan for ES already exists, it remains an invasive tumor with a high recurrence rate and a low 5-year survival rate (2). Previous studies have revealed that the EWS-ETS fusion gene may be the driving gene for ES; however, targeted therapy for the EWS-ETS fusion gene is still difficult to achieve (2). Therefore, it is necessary to identify new target genes, which may lead to the improvement of prognosis in patients with ES.

In the present study, two modules associated with OVS and metastatic state were identified by weighted co-expression analysis. The GO and KEGG pathway enrichment analysis of the OVS-associated module revealed that the genes in this module were enriched in the immune response and cell cycle, including terms such as 'adaptive immune system' and 'pathways in cancer'. The results of the WGCNA analysis demonstrated that PSMA4 served a hub role in the PPI network of the OVS-associated module. Previous studies have reported that mutations in PSMA4 may be associated with familial lung cancer $(19,20)$, which suggests that PSMA4 may be a potential proto-oncogene. In addition, PSMA4 serves a role in chemotherapy resistance and immune response (21). PSMA4 forms a complex with proteasome activator subunits 3 and 4, which is required in the processing of major histocompatibility complex (MHC) class I-presented antigenic peptides (22-24). MHC class I serve a crucial role in the activation of cytotoxic T-cells, which is responsible for the tumor-associated immune response $(25,26)$. Therefore, PSMA4 may be associated with tumor immune disorders, and further research is needed to elucidate the role of PSMA4 in the carcinogenesis of ES.

The protein encoded by L1CAM is an axonal glycoprotein that belongs to the immunoglobulin supergene family. L1CAM is a cell adhesion molecule associated with the prognosis of ovarian cancer, melanoma and endometrial cancer (27-29), and multivariate Cox survival analysis has suggested that L1CAM may be an independent prognostic variable in a number of different types of cancer, although there were no studies examining it potential as a prognostic variable in ES (30). These results suggest that L1CAM may serve a crucial role in tumor progression. In addition to that in cancerous tissues, abnormal L1CAM expression has also been identified in precancerous lesions. Geismann et al (31) revealed the role of L1CAM in the malignant transformation of pancreatic cancer, and an association with precancerous lesions was also identified in inflammatory bowel disease (32) and endometriosis (33). Taken together, previous studies have demonstrated the crucial role of L1CAM in the prognosis of carcinomas originating from epithelial tissue. The results of the present study revealed that L1CAM may also be a potential prognostic biomarker of ES. 


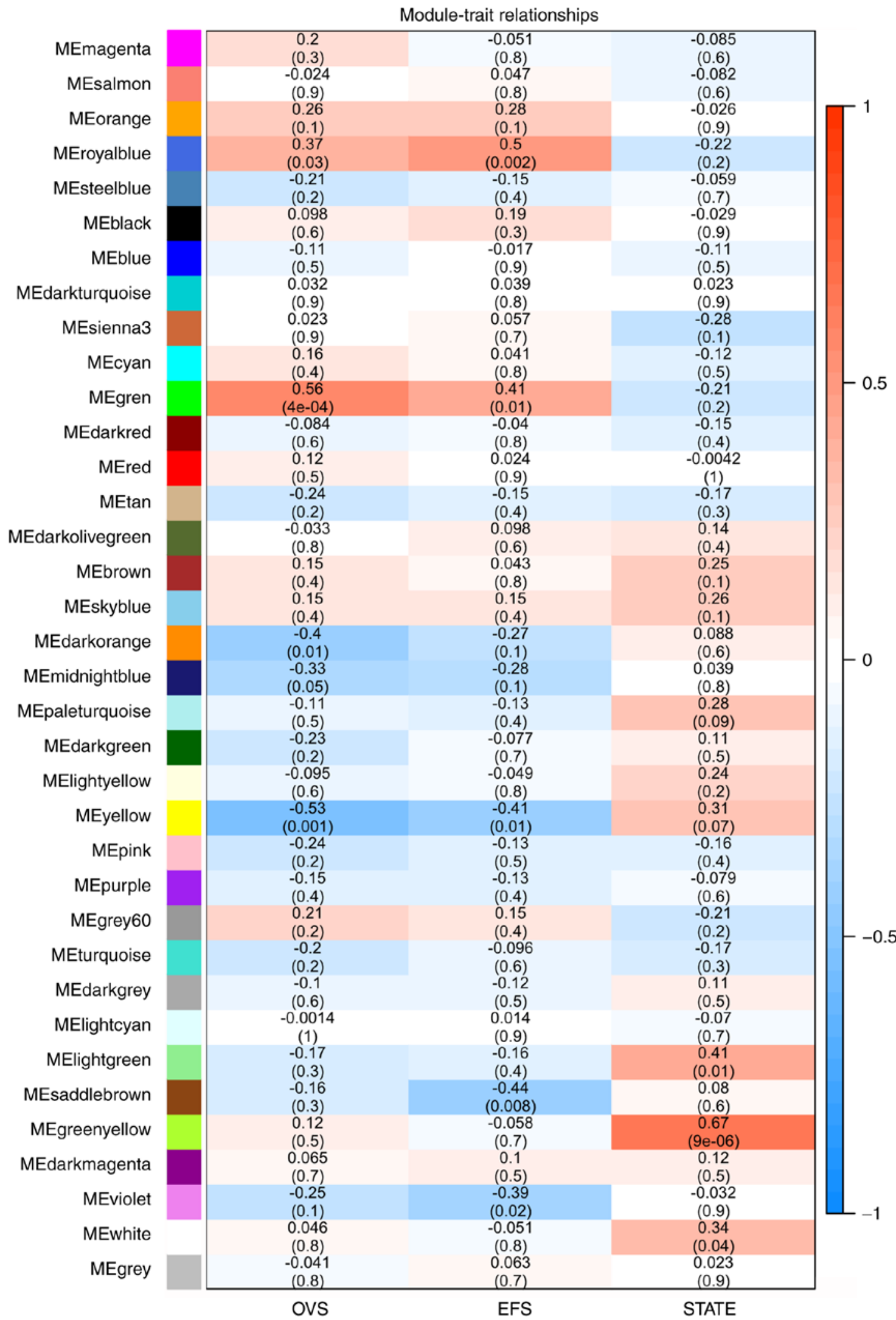

Figure 4. Heatmap of the correlation between identified modules and clinical features. The x-axis corresponds to clinical features, and the y-axis represents the identified modules. The color scale (blue to red) indicates correlation. Data are presented as the correlation coefficient in the top row and the P-value in the bottom row. OVS, overall vital survival; EFS, event-free survival; STATE, metastatic state.

In a previous study, STRAP was described to negatively regulate the transforming growth factor $\mathrm{b}$ (TGF- $\beta$ ) signaling pathway by binding to Smad7 (34). STRAP regulates the mitogen-activated protein kinase/extracellular signal-regulated kinase signaling pathway in a unmber ofdifferent types of tumor (35). Overexpression of STRAP has been identified in osteosarcoma (36). In addition, downstream of STRAP, the TGF- $\beta$ signaling pathway is a crucial osteoblastic signaling 
Table I. Top 6 clusters with representative enriched terms (one per cluster).

\begin{tabular}{|c|c|c|c|c|}
\hline Category & Description & $\%^{\mathrm{a}}$ & $\log 10(P)^{b}$ & Genes \\
\hline R-HSA-1280218 & Adaptive immune system & 30 & -10.45 & $\begin{array}{l}\text { CLTA, DYNC1I2, FKBP1A, PDPK1, PRKCB, } \\
\text { PSMA4, FBXW4, SKP1, TRAF6, UBE2H, } \\
\text { RBX1, HERC5, UBA6, TUBA1C, CDC } 26\end{array}$ \\
\hline hsa05200 & Pathways in cancer & 20 & -7.99 & $\begin{array}{l}\text { CCND1, FZD2, GNG5, JAK1, PRKCB, RXRA, } \\
\text { TGFBR1, TRAF3, TRAF6, RBX1 }\end{array}$ \\
\hline R-HSA-69278 & Cell cycle, mitotic & 22 & -7.81 & $\begin{array}{l}\text { CCND1, CCND3, CCNH, DYNC1I2, PRKCB, } \\
\text { PSMA4, SKP1, LPIN2, RBX1, TUBA1C, } \\
\text { CDC26 }\end{array}$ \\
\hline M-286 & PID TGFBR PATHWAY ${ }^{c}$ & 10 & -6.92 & FKBP1A, PDPK1, PPP1CA, TGFBR1, STRAP \\
\hline R-HSA-373760 & L1CAM interactions & 12 & -6.67 & $\begin{array}{l}\text { ANK1, CLTA, EPHB2, L1CAM, NUMB, } \\
\text { TUBA1C }\end{array}$ \\
\hline hsa04110 & Cell cycle & 12 & -6.57 & $\begin{array}{l}\text { CCND1, CCND3, CCNH, SKP1, RBX1, } \\
\text { CDC26 }\end{array}$ \\
\hline
\end{tabular}

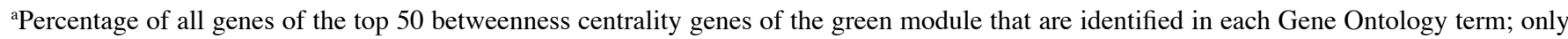

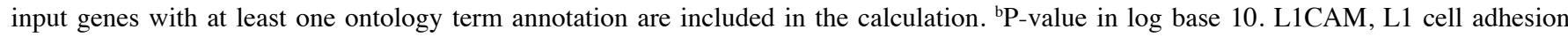

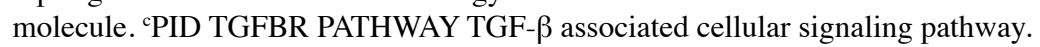

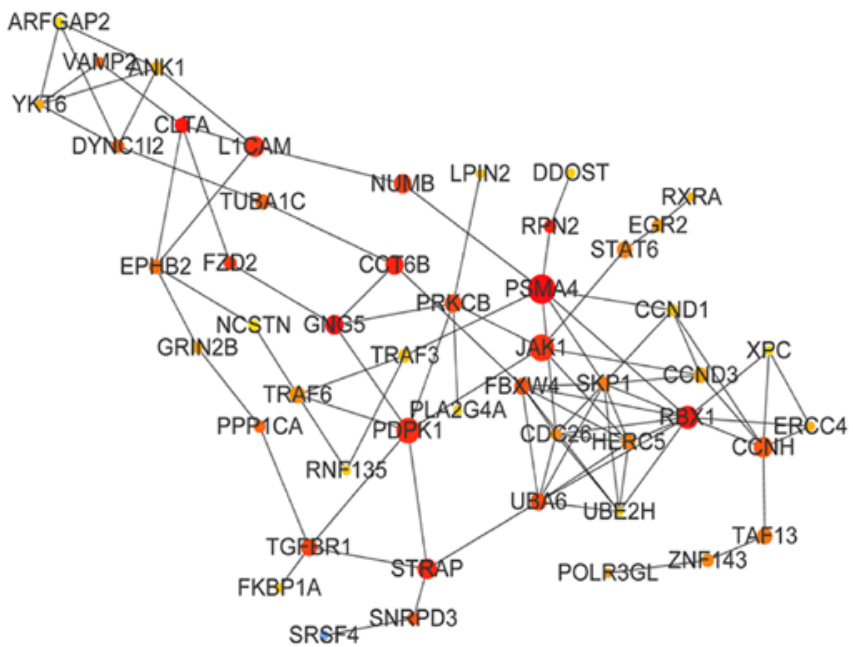

Figure 5. Protein-protein interaction network of the top 50 high-BC genes in the overall vital survival-associated module. Nodes of the network represent proteins; the size of each node represents the BC of the node. Lines represent the interactions between the proteins according to the STRING database. $\mathrm{BC}$, betweenness centrality.

pathway in the early stage of bone formation (37), and has been identified to be overexpressed in high-grade osteosarcoma tissue (38). Since STRAP serves osteogenic and oncogenic roles in bone tumors, further evidence is needed to determine the role of STRAP in the tumorigenesis of ES.

In the present study, KEGG pathway and GO functional enrichment showed that the top $50 \mathrm{BC}$ genes in the metastasis-associated module were mostly enriched in terms associated with metabolism and signaling pathways. According to the literature, CACNG2 encodes a protein involved in the trafficking of glutamatergic AMPA receptors (39). Previous studies have revealed the key role of CACNG2 in mental and neuropathic

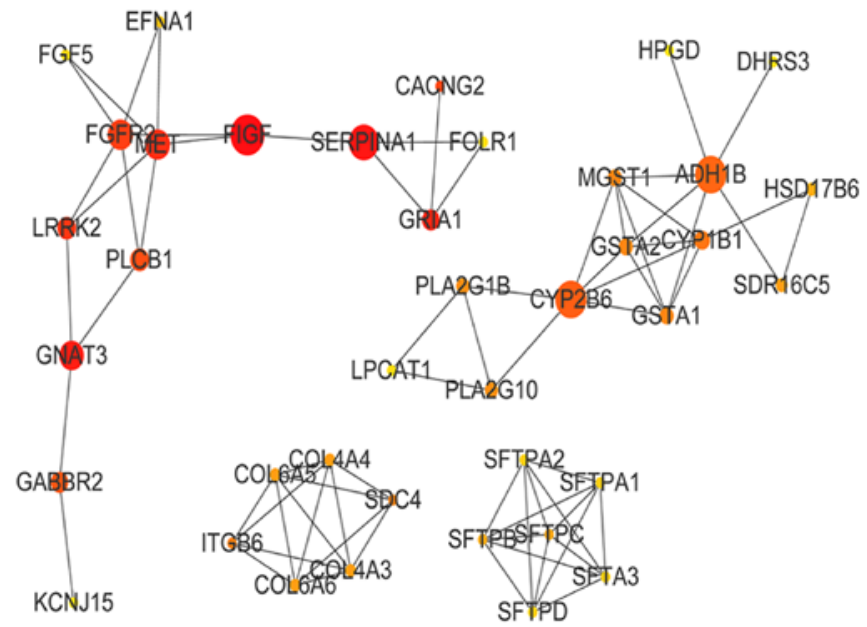

Figure 6. Protein-protein interaction network of the top 50 high-BC genes in the metastasis-associated module. Nodes of the network represent proteins; the size of each node represents the BC of the node. Lines represent the interactions between the proteins according to the Search Tool for the Retrieval of Interacting Genes/Proteins database. BC, betweenness centrality.

disorders, including bipolar disorder, schizophrenia (40) and chronic pain (39). GABBR2 is a protein involved in neurological disorders, such as epilepsy (41) and Huntington's disease (42). However, the roles of CACNG2 and GABBR2 in ES are still unclear. The results of the present study suggested that these two genes may serve as potential biomarkers for metastasis or prognosis in patients with ES. Further research is needed to elucidate their role in this disease.

In conclusion, the present study identified several potential molecules involved in the metastasis and prognosis of ES. The WGCNA analysis identified certain co-expression modules in ES, which were associated with clinical features 
Table II. Top 6 clusters with representative enriched terms (one per cluster).

\begin{tabular}{|c|c|c|c|c|}
\hline GO & Description & $\%^{\mathrm{a}}$ & $\log 10(\mathrm{P})^{\mathrm{b}}$ & Genes \\
\hline R-HSA-5688849 & $\begin{array}{l}\text { Defective CSF2RB causes } \\
\text { pulmonary surfactant metabolism } \\
\text { dysfunction } 5 \text { (SMDP5) }\end{array}$ & 12 & -14.69 & $\begin{array}{l}\text { SFTPB, SFTPC, SFTPD, SFTA3, } \\
\text { SFTPA1, SFTPA2 }\end{array}$ \\
\hline hsa04151 & PI3K-Akt signaling pathway & 20 & -8.59 & $\begin{array}{llll}\text { COL4A3, COL4A4, } & \text { EFNA1, } & \text { FGF5 } \\
\text { FGFR2, } & \text { VEGFD, } & \text { ITGB6, } & \text { MET } \\
\text { COL6A6, COL6A5 } & & \end{array}$ \\
\hline hsa00980 & $\begin{array}{l}\text { Metabolism of xenobiotics } \\
\text { by cytochrome } \mathrm{P} 450\end{array}$ & 12 & -7.91 & $\begin{array}{l}\text { ADH1B, CYP1B1, CYP2B6, GSTA1, } \\
\text { GSTA2, MGST1 }\end{array}$ \\
\hline hsa04014 & Ras signaling pathway & 14 & -6.26 & $\begin{array}{l}\text { EFNA1, FGF5, FGFR2, VEGFD, MET } \\
\text { PLA2G1B, PLA2G10 }\end{array}$ \\
\hline hsa00830 & Retinol metabolism & 8 & -4.93 & ADH1B, CYP2B6, HSD17B6, SDR16C5 \\
\hline R-HSA-112314 & $\begin{array}{l}\text { Neurotransmitter receptors and } \\
\text { postsynaptic signal transmission }\end{array}$ & 10 & -4.68 & $\begin{array}{l}\text { GRIA1, GABBR2, CACNG2, PLCB1, } \\
\text { GNAT3 }\end{array}$ \\
\hline
\end{tabular}

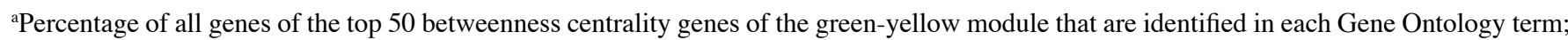
only input genes with at least one ontology term annotation are included in the calculation. ${ }^{\mathrm{b}} \mathrm{P}$-value in $\log$ base 10 . CSF2RB, colony-stimulating factor 2 receptor $\beta$ common subunit.
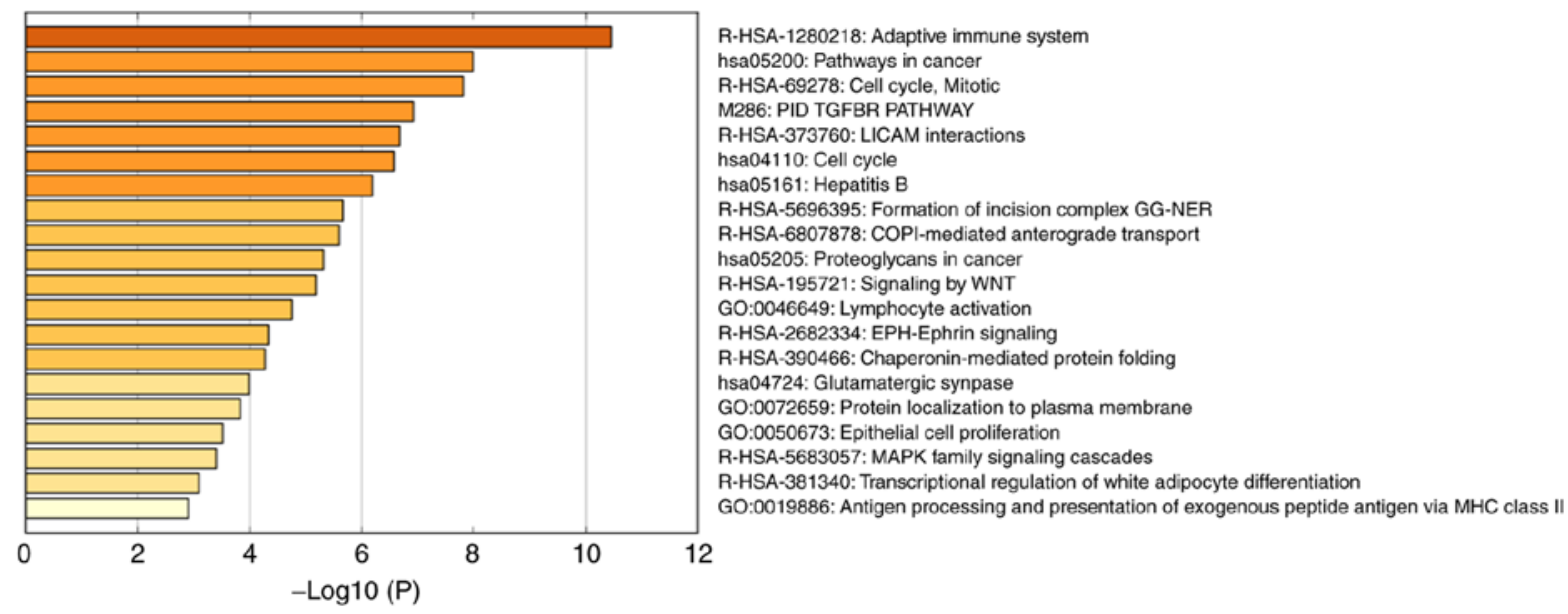

$-\log 10(P)$

Figure 7. Heatmap of enriched terms across the top 50 high-betweenness centrality genes across the gene lists of the overall vital survival associated module.

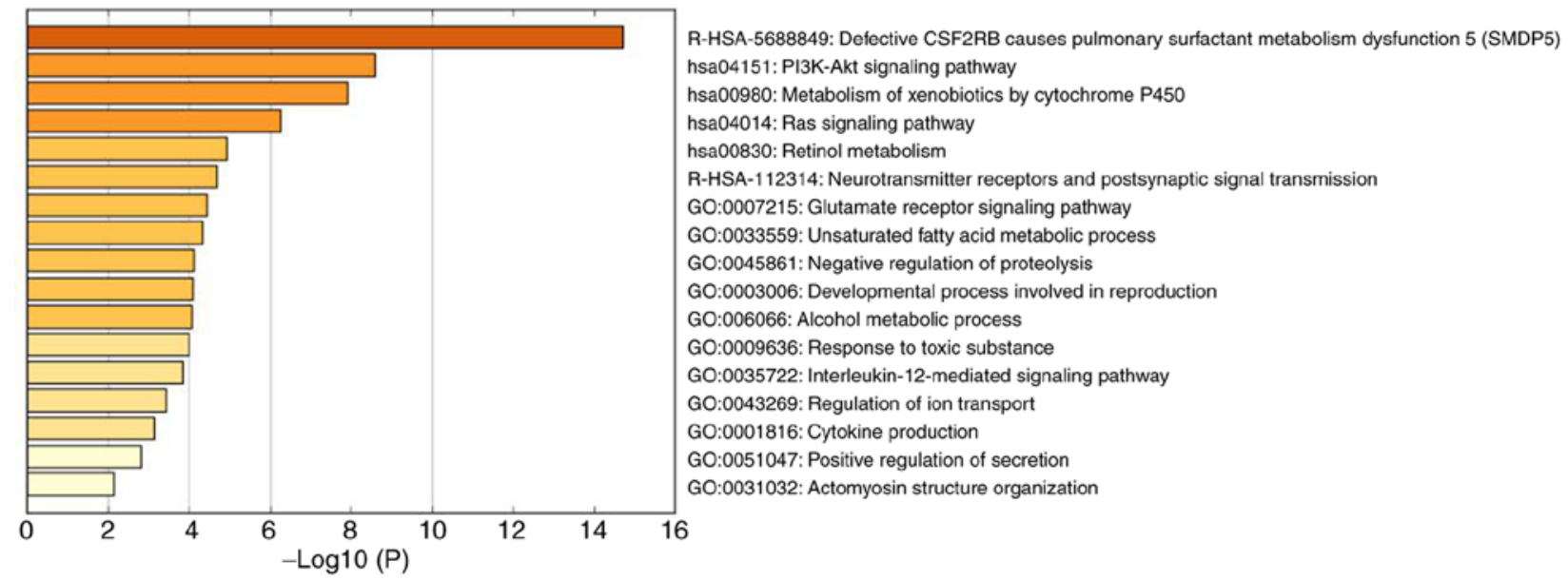

Figure 8. Heatmap of enriched terms across the gene lists of the metastasis-associated module, colored according to P-value. 

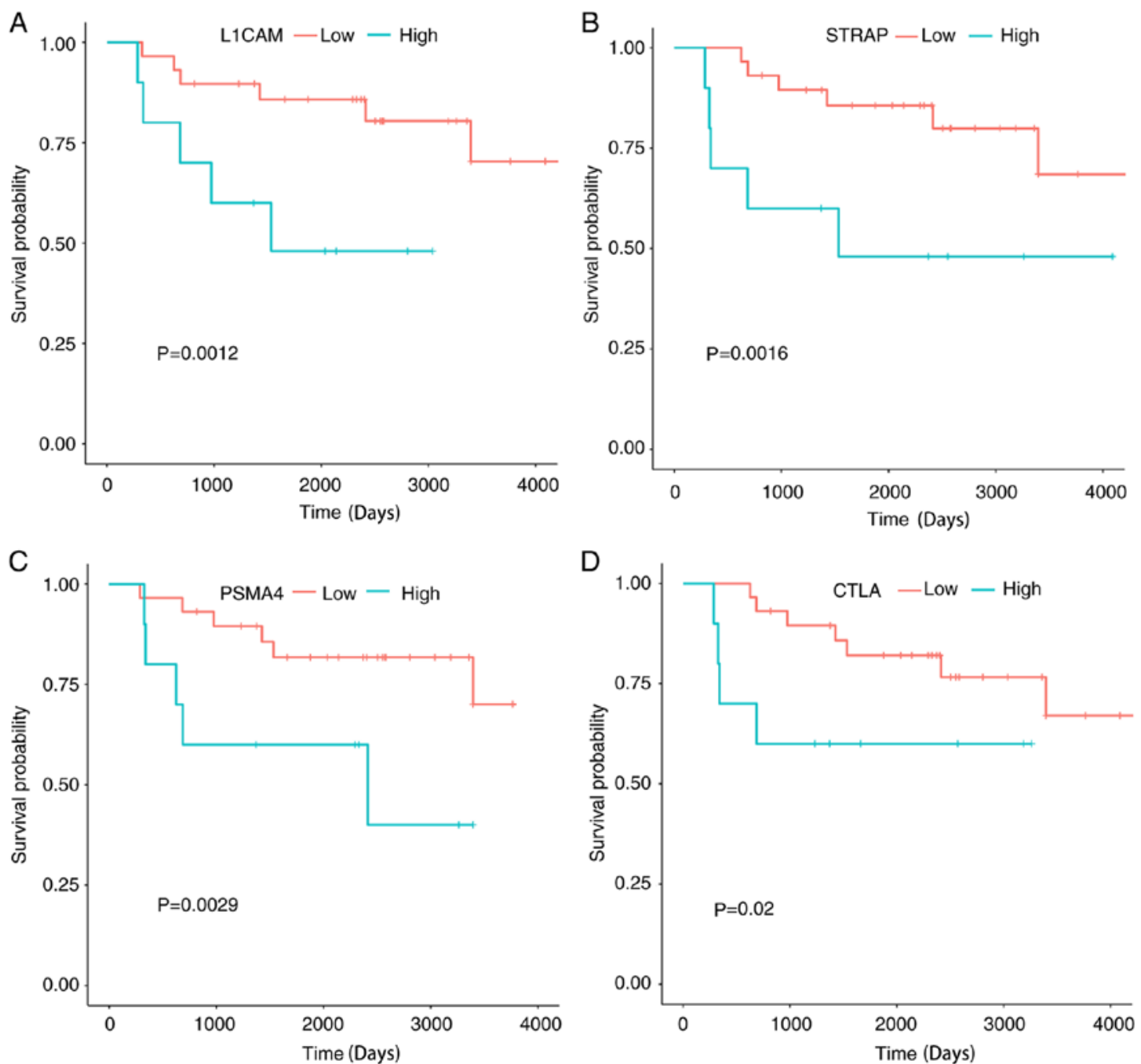

Figure 9. Survival curve of the top 4 significant genes in the overall vital survival-associated module. (A) L1CAM, L1 cell adhesion molecule; (B) STRAP, serine/threonine kinase receptor-associated protein; (C) PSMA4, proteasome subunit $\alpha 4$; (D) CTLA, cytotoxic T-lymphocyte-associated protein 4.
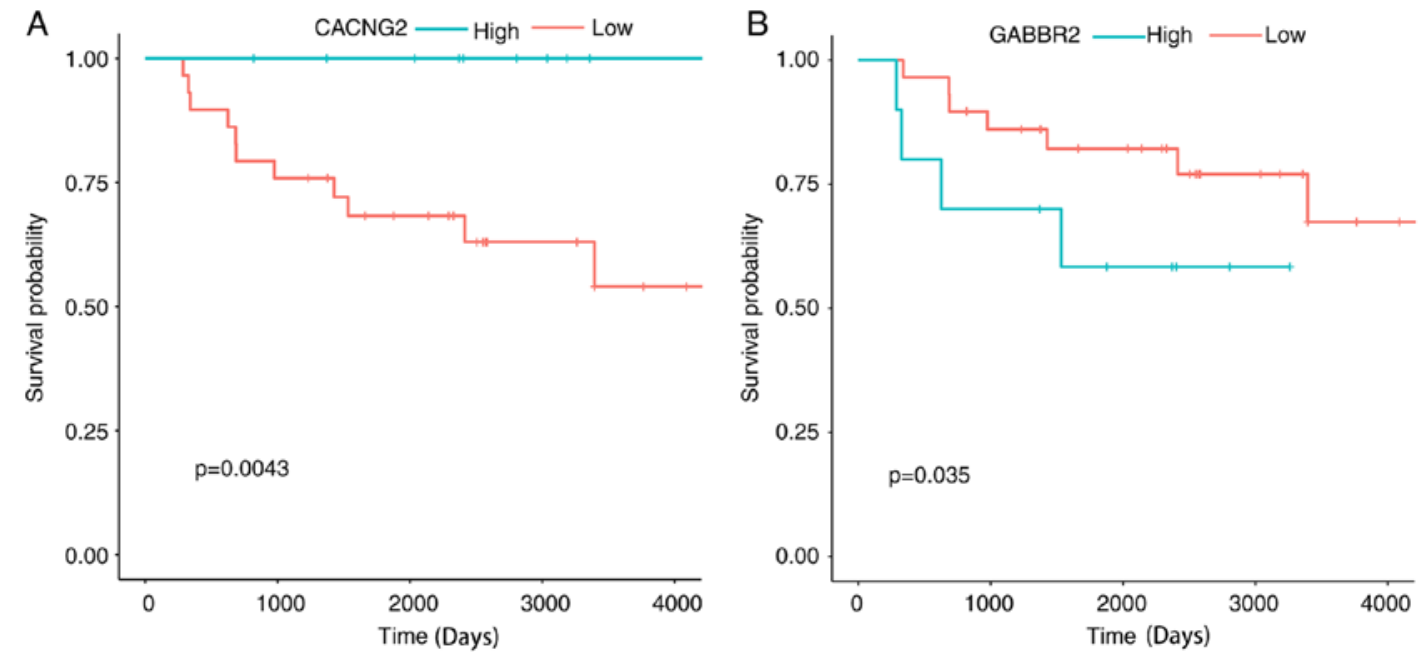

Figure 10. Survival curves of (A) CACNG2 and (B) GABBR2. Genes in the metastasis-associated module were also associated with the overall vital survival of patients with Ewing sarcoma. CACNG2, calcium voltage-gated channel auxiliary subunit $\gamma 2$; GABR2, $\gamma$-aminobutyric acid type B receptor subunit 2 .

of the samples, to screen for clinical feature-associated genes. The hub genes identified in the present study require further research due to the lack of associated studies. The
PPI network and functional enrichment of the hub genes may provide a view into the gene co-expression interaction framework of ES. 


\section{Acknowledgements}

Not applicable.

\section{Funding}

No funding was received.

\section{Availability of data and materials}

The datasets generated and/or analyzed during the current study are available in the GEO repository,(accession nos. GSE63157 and GSE17679).

\section{Authors' contributions}

BW, JL and XL analyzed the data and wrote the manuscript. YO designed the experiment and revised the manuscript. The final version of the manuscript has been approved by all authors.

\section{Ethics approval and consent to participate}

Not applicable.

\section{Patient consent for publication}

Not applicable.

\section{Competing interests}

The authors declare that they have no competing interests.

\section{References}

1. Whelan J, McTiernan A, Cooper N, Wong YK, Francis M, Vernon S and Strauss SJ: Incidence and survival of malignant bone sarcomas in England 1979-2007. Int J Cancer 131: E508-E517, 2012.

2. Grunewald TGP, Cidre-Aranaz F, Surdez D, Tomazou EM, de Álava E, Kovar H, Sorensen PH, Delattre O and Dirksen U: Ewing sarcoma. Nat Rev Dis Primers 4: 5, 2018.

3. Stahl M, Ranft A, Paulussen M, Bölling T, Vieth V, Bielack S, Görtitz I, Braun-Munzinger G, Hardes J, Jürgens $H$ and Dirksen U: Risk of recurrence and survival after relapse in patients with Ewing sarcoma. Pediatr Blood Cancer 57: 549-553, 2011.

4. Burchill SA: Ewing's sarcoma: Diagnostic, prognostic, and therapeutic implications of molecular abnormalities. J Clin Pathol 56: 96-102, 2003.

5. Riggi N, Knoechel B, Gillespie SM, Rheinbay E, Boulay G, Suvà $\mathrm{ML}$, Rossetti $\mathrm{NE}$, Boonseng $\mathrm{WE}, \mathrm{Oksuz} \mathrm{O}, \mathrm{Cook} \mathrm{EB}$, et al: EWS-FLI1 utilizes divergent chromatin remodeling mechanisms to directly activate or repress enhancer elements in Ewing sarcoma. Cancer Cell 26: 668-681, 2014.

6. Sankar S, Bell R, Stephens B, Zhuo R, Sharma S, Bearss DJ and Lessnick SL: Mechanism and relevance of EWS/FLI-mediated transcriptional repression in Ewing sarcoma. Oncogene 32: 5089-5100, 2013.

7. Chaturvedi A, Hoffman LM, Welm AL, Lessnick SL and Beckerle MC: The EWS/FLI oncogene drives changes in cellular morphology, adhesion, and migration in ewing sarcoma. Genes Cancer 3: 102-116, 2012.

8. Fadul J, Bell R, Hoffman LM, Beckerle MC, Engel ME and Lessnick SL: EWS/FLI utilizes NKX2-2 to repress mesenchymal features of Ewing sarcoma. Genes Cancer 6: 129-143, 2015.
9. Pedersen EA, Menon R, Bailey KM, Thomas DG, Van Noord RA, Tran J, Wang H, Qu PP, Hoering A, Fearon ER, et al: Activation of Wnt/ $\beta$-catenin in ewing sarcoma cells antagonizes EWS/ETS function and promotes phenotypic transition to more metastatic cell states. Cancer Res 76: 5040-5053, 2016.

10. de Alava E, Kawai A, Healey JH,Fligman I, Meyers PA, Huvos AG, Gerald WL, Jhanwar SC, Argani P, Antonescu CR, et al: EWS-FLI1 fusion transcript structure is an independent determinant of prognosis in Ewing's sarcoma. J Clin Oncol 16: 1248-1255, 1998.

11. Le Deley MC, Delattre O, Schaefer KL, Burchill SA, Koehler G, Hogendoorn PC, Lion T, Poremba C, Marandet J, Ballet S, et al: Impact of EWS-ETS fusion type on disease progression in Ewing's sarcoma/peripheral primitive neuroectodermal tumor: Prospective results from the cooperative Euro-E.W.I.N.G. 99 trial. J Clin Oncol 28: 1982-1988, 2010.

12. van Doorninck JA, Ji L, Schaub B, Shimada H, Wing MR, Krailo MD, Lessnick SL, Marina N, Triche TJ, Sposto R, et al: Current treatment protocols have eliminated the prognostic advantage of type 1 fusions in Ewing sarcoma: A report from the Children's Oncology Group. J Clin Oncol 28: 1989-1994, 2010.

13. Langfelder P and Horvath S: WGCNA: An R package for weighted correlation network analysis. BMC Bioinformatics 9: $559,2008$.

14. Saris CG, Horvath S, van Vught PW, van Es MA, Blauw HM, Fuller TF, Langfelder P, DeYoung J, Wokke JH, Veldink JH, et al: Weighted gene co-expression network analysis of the peripheral blood from Amyotrophic Lateral Sclerosis patients. BMC Genomics 10: 405, 2009.

15. Savola S, Klami A, Myllykangas S, Manara C, Scotlandi K, Picci P, Knuutila $S$ and Vakkila J: High expression of complement component 5 (C5) at tumor site associates with superior survival in Ewing's sarcoma family of tumour patients. ISRN Oncol 2011: 168712, 2011.

16. M K: Post-genome informatics, 2000.

17. Volchenboum SL, Andrade J, Huang L, Barkauskas DA, Krailo M, Womer RB, Ranft A, Potratz J, Dirksen U, Triche TJ and Lawlor ER: Gene expression profiling of ewing sarcoma tumors reveals the prognostic importance of tumor-stromal interactions: A report from the Children's oncology group. J Pathol Clin Res 1: 83-94, 2015.

18. Kovar H, Dworzak M, Strehl S, Schnell E, Ambros IM, Ambros PF and Gadner H: Overexpression of the pseudoautosomal gene MIC2 in Ewing's sarcoma and peripheral primitive neuroectodermal tumor. Oncogene 5: 1067-1070, 1990.

19. Amos CI, Wu X, Broderick P, Gorlov IP, Gu J, Eisen T, Dong Q, Zhang Q, Gu X, Vijayakrishnan J, et al: Genome-wide association scan of tag SNPs identifies a susceptibility locus for lung cancer at 15q25.1. Nat Genet 40: 616-622, 2008.

20. Liu P, Vikis HG, Wang D, Lu Y, Wang Y, Schwartz AG, Pinney SM, Yang P, de Andrade M, Petersen GM, et al: Familial aggregation of common sequence variants on 15q24-25.1 in lung cancer. J Natl Cancer Inst 100: 1326-1330, 2008.

21. Wang T, Chen T, Thakur A, Liang Y, Gao L, Zhang S, Tian Y, Jin T, Liu JJ and Chen M: Association of PSMA4 polymorphisms with lung cancer susceptibility and response to cisplatin-based chemotherapy in a Chinese Han population. Clin Transl Oncol 17: 564-569, 2015.

22. Groettrup M, Soza A, Eggers M, Kuehn L, Dick TP, Schild H, Rammensee HG, Koszinowski UH and Kloetzel PM: A role for the proteasome regulator PA28alpha in antigen presentation. Nature 381: 166-168, 1996.

23. Rut W and Drag M: Human 20S proteasome activity towards fluorogenic peptides of various chain lengths. Biol Chem 397: 921-926, 2016.

24. Yano M, Koumoto Y, Kanesaki Y, Wu X and Kido H: 20S proteasome prevents aggregation of heat-denatured proteins without PA700 regulatory subcomplex like a molecular chaperone. Biomacromolecules 5: 1465-1469, 2004.

25. Rodig SJ, Gusenleitner D,Jackson DG, Gjini E, Giobbie-Hurder A Jin C, Chang H, Lovitch SB, Horak C, Weber JS, et al: MHC proteins confer differential sensitivity to CTLA-4 and PD-1 blockade in untreated metastatic melanoma. Sci Transl Med 10: pii: eaar3342, 2018.

26. Nakayama M: Antigen presentation by MHC-dressed cells. Front Immunol 5: 672, 2014.

27. Fogel M, Gutwein P, Mechtersheimer S, Riedle S, Stoeck A, Smirnov A, Edler L, Ben-Arie A, Huszar M and Altevogt P: L1 expression as a predictor of progression and survival in patients with uterine and ovarian carcinomas. Lancet 362: 869-875, 2003. 
28. Fogel M, Mechtersheimer S, Huszar M, Smirnov A, Abu-Dahi A, Tilgen W, Reichrath J, Georg T, Altevogt P and Gutwein P: L1 adhesion molecule (CD 171) in development and progression of human malignant melanoma. Cancer Lett 189: 237-247, 2003.

29. Zeimet AG, Reimer D, Huszar M, Winterhoff B, Puistola U, Azim SA, Müller-Holzner E, Ben-Arie A, van Kempen LC, Petru E, et al: L1CAM in early-stage type I endometrial cancer: Results of a large multicenter evaluation. J Natl Cancer Inst 105 1142-1150, 2013.

30. Altevogt P, Doberstein K and Fogel M: L1CAM in human cancer. Int J Cancer 138: 1565-1576, 2016.

31. Geismann C, Morscheck M, Koch D, Bergmann F, Ungefroren $H$, Arlt A, Tsao MS, Bachem MG, Altevogt P, Sipos B, et al: Up-regulation of L1CAM in pancreatic duct cells is transforming growth factor beta1- and slug-dependent: Role in malignant transformation of pancreatic cancer. Cancer Res 69: 4517-4526, 2009.

32. Schafer H, Struck B, Feldmann EM, Bergmann F, GrageGriebenow E, Geismann C, Ehlers S, Altevogt P and Sebens S TGF- $\beta 1$-dependent L1CAM expression has an essential role in macrophage-induced apoptosis resistance and cell migration of human intestinal epithelial cells. Oncogene 32: 180-189, 2013.

33. Finas D, Huszar M, Agic A, Dogan S, Kiefel H, Riedle S, Gast D, Marcovich R, Noack F, Altevogt P, et al: L1 cell adhesion molecule (L1CAM) as a pathogenetic factor in endometriosis. Hum Reprod 23: 1053-1062, 2008.

34. Datta PK and Moses HL: STRAP and Smad7 synergize in the inhibition of transforming growth factor beta signaling. Mol Cell Biol 20: 3157-3167, 2000

35. Halder SK, Anumanthan G, Maddula R, Mann J, Chytil A, Gonzalez AL, Washington MK, Moses HL, Beauchamp RD and Datta PK: Oncogenic function of a novel WD-domain protein, STRAP, in human carcinogenesis. Cancer Res 66: 6156-6166, 2006.
36. Pruksakorn D, Klangjorhor J, Lirdprapamongkol K, Teeyakasem P, Sungngam P, Chaiyawat P, Phanphaisarn A, Settakorn J and Srisomsap C: Oncogenic roles of serine-threonine kinase receptor-associated protein (STRAP) in osteosarcoma. Cancer Chemother Pharmacol 82: 1039-1047, 2018.

37. Chen G, Deng C and Li YP: TGF- $\beta$ and BMP signaling in osteoblast differentiation and bone formation. Int J Biol Sci 8: 272-288, 2012

38. Franchi A, Arganini L, Baroni G, Calzolari A, Capanna R, Campanacci D, Caldora P, Masi L, Brandi ML and Zampi G: Expression of transforming growth factor beta isoforms in osteosarcoma variants: Association of TGF beta 1 with high-grade osteosarcomas. J Pathol 185: 284-289, 1998.

39. Nissenbaum J, Devor M, Seltzer Z, Gebauer M, Michaelis M, Tal M, Dorfman R, Abitbul-Yarkoni M, Lu Y, Elahipanah T, et al: Susceptibility to chronic pain following nerve injury is genetically affected by CACNG2. Genome Res 20: 1180-1190, 2010.

40. Liu YL, Fann CS, Liu CM, Chen WJ, Wu JY, Hung SI, Chen CH, Jou YS, Liu SK, Hwang TJ, et al: RASD2, MYH9, and CACNG2 genes at chromosome $22 \mathrm{q} 12$ associated with the subgroup of schizophrenia with non-deficit in sustained attention and executive function. Biol Psychiatry 64: 789-796, 2008.

41. Yoo Y, Jung J, Lee YN, Lee Y, Cho H, Na E, Hong J, Kim E, Lee JS, Lee JS, et al: GABBR2 mutations determine phenotype in rett syndrome and epileptic encephalopathy. Ann Neurol 82: 466-478, 2017.

42. Philpott AL, Fitzgerald PB, Bailey NW, Churchyard A, Georgiou-Karistianis N and Cummins TD: A GABBR2 gene variant modifies pathophysiology in Huntington's disease. Neurosci Lett 620: 8-13, 2016.

This work is licensed under a Creative Commons Attribution-NonCommercial-NoDerivatives 4.0 International (CC BY-NC-ND 4.0) License. 